\title{
NEW CAPABILITIES OF THE SPIN TRACKING CODE SPINK*
}

\author{
A.Luccio", A.Lehrach, J.Niederer, T.Roser, M.Syphers, and N.Tsoupas, BNL, Upton, NY
}

\section{Abstract}

The code $S P I N K$, originally written to track polarized protons in RHIC, underwent several modifications and additions that have added substantial new capabilities to it.

\section{BASIC FEATURES OF SPINK}

A tracking code: $S P I N K$ [1] was written for the RHIC SPIN project at Brookhaven [2] to study the behavior of polarized protons in the Relativistic Collider during injection, acceleration and storage at fixed energy. From the output of MAD [3] SPINK reads the first order matrices for each machine element and the second order Transport maps as well as the Twiss functions along the lattice. Then, a subset of matrices is being created, where all elements that do not generate any spin rotation or any bend in the trajectory are lumped together. To the newly created elements a keyword is attached, such as BEND, QUAD, SEXT, HELIX, SNAKE, RFCAV, etc. Particle orbits are tracked through the first and second order maps (thick elements, with edge effects, as described in $M A D$ ). The spin of each proton, represented by a real 3dimensional vector of length one, is also tracked using spin rotation matrices created by the code. At the present, with the exception of Helices, spin rotation matrices are for thin elements.

SPINK has been used for years, both in RHIC and the AGS, in particular to study the survival of the spin polarization in passing through resonances. The most significant new features of the code are described below.

\section{LATTICE ERRORS}

Angle, position and field errors in the machine lattice generate a distorted closed orbit. Since the strength of spin resonances depends on the average distance of a particle from the design equilibrium orbit, depolarization at a resonance is affected by lattice errors. In an actual accelerator the closed orbit distortions are being corrected with the use of horizontal and vertical steering magnets, whose currents are calculated with various algorithms. Orbit correction in MAD is accomplished through the Micado algorithm, that solves in the least square approximation an under constrained system of equations.

To correctly track phase space and spin, we had to transmit from $M A D$ to $S P I N K$ the information related to the errors (measured or randomly assumed) of each machine element, and displace and rotate accordingly the relevant orbit transport maps. This task is accomplished using the BNL version of MAD [4].

Results of proton spin tracking in the RHIC lattice in the presence of errors for two intrinsic resonances, for an ideal orbit (no lattice errors), and for errors in the lattice but with a corrected orbit (to $0.2 \mathrm{~mm}$ ), are shown in
Fig.1. Here, we assumed random errors of the order of 1 $\mathrm{mm}$ and $1 \mathrm{mrad}$ in position and angle of all magnets, respectively. The momentum spread in the beam was $0.03 \%$.

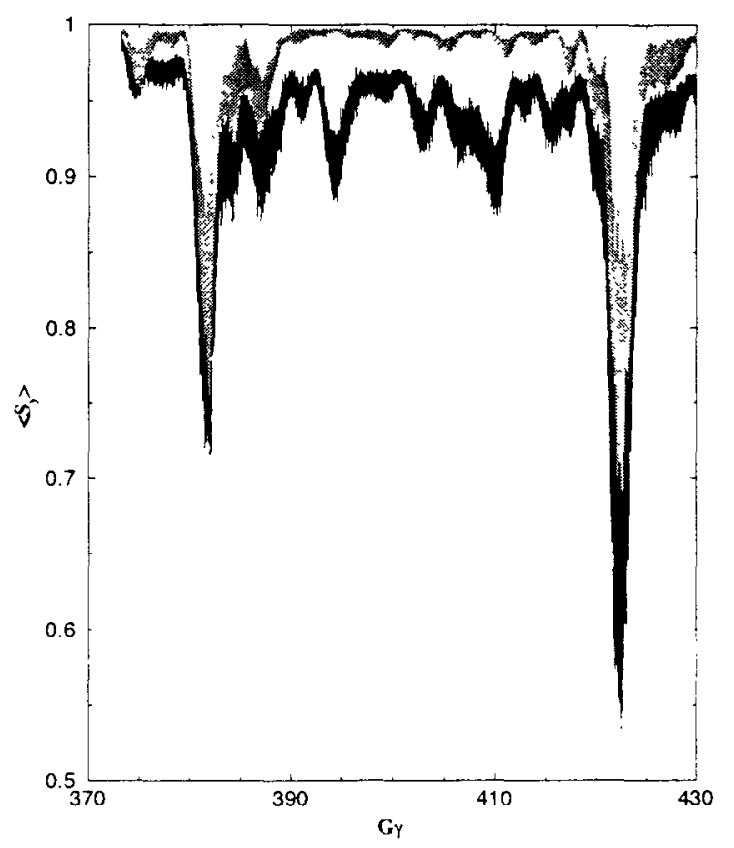

Figure 1: Spin tracking in RHIC, crossing the $\mathrm{G} \gamma=$ 381.82 and 422.18 intrinsic resonances. The upper curve is for an ideal orbit. The lower, for a distorted orbit due to lattice errors. Average over a few hundred particles tracked.

\section{SECOND ORDER EFFECTS}

Second order effects in SPINK are dealt with using the second order Transport maps from $M A D$. Important effects on spin resonances arise from crossing of sextupoles and higher order multipoles in the lattice.

Since in building maps for orbit tracking some elements are lumped together as explained in Sec. 1, matrices have to be multiplied, and also second order maps are convoluted to produce an overall map for the new element. This is done using the expression

$$
T_{i j k}=\sum_{n=1}^{6}\left(R_{i n}^{\prime \prime} T_{n j k}^{\prime}+\sum_{m=1}^{6} T_{t n m}^{\prime \prime} R_{r j}^{\prime} R_{m k}^{\prime}\right)
$$

Where $R$ and $T$ are the first order and the second order orbit Transport maps, respectively, and the index (") or (") denotes the order in which maps are taken.

\footnotetext{
"Work supported by the U.S. Department of Energy

"luccio@bni.gov
} 
Two comments are necessary: (i) Second order tracking considerably reduces the speed of calculation, which is an unpleasant factor when many particles for many turns are tracked. (ii) Second order Transport maps are truncated and therefore non-simplectic.

In conclusion, in performing second order tracking one has to exercise caution when the conservation of quantities like the emittance is an important issue. Using of better maps, like the one derived with Lie algebra will be implemented in the future.

\section{BEAM-BEAM EFFECTS}

In RHIC, the two acceleration chambers intersect at six locations around the circumference, where the counter rotating beams can be brought to collide. Collisions affect both orbit and spin dynamics, since the electric and magnetic field of a beam exercise forces on the particles of the other beam, producing defocusing and spin rotation.

In $S P I N K$, we modeled one beam as a Gaussian distribution of charges in motion and we studied the effect of the fields on the single particles of the other beam [5]. The expression of the resulting betatron tune shift in a collision is

$$
\Delta v=-\frac{\xi}{u^{2}}\left[1-e^{-u^{\prime}} I_{0}\left(u^{2}\right)\right]
$$

where $u=r / 2 \sigma$, and $\xi$ is the tune shift on axis, proportional to the number of particles per bunch $n$ and inversely to the normalized emittance. E.g. for $n=$ $2 \times 10^{11}$, emittance $10 \pi \mathrm{mm}-\mathrm{mrad}$ and $\beta^{*}=1 \mathrm{~m}$, it is on axis $\xi=-0.015$. Tune distribution in the beam can be calculated by $\mathrm{Eq}$. (2). SPINK tracking agreed very well with Eq. (2) and was also used to calculate the effect of beam-beam on spin. Fig. 3 shows how the vertical component of the spin is affected by beam-beam at fixed energy corresponding to $G \gamma=261$, close to the strong intrinsic spin resonance at $G \gamma=232+v_{\mathrm{y}}$.

For this simulation run, the beams are first accelerated. then brought to constant energy and made to collide.

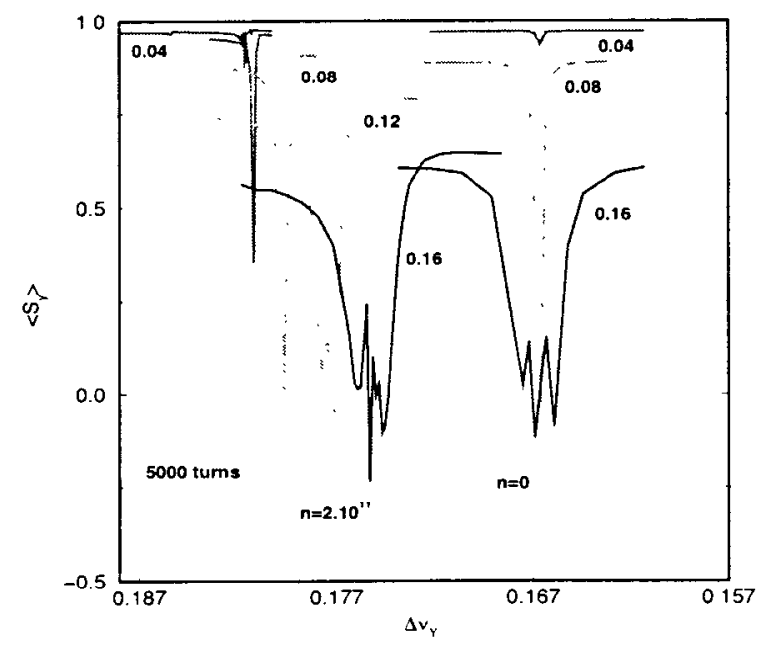

Figure 2: Beam-beam in a resonance crossing. The tune of particles at various vertical distances from axis determines the resonance strength. No space charge, and $n=2 \times 10^{11}$.

\section{STABLE SPIN AXIS. SPIN TUNE}

For each position along the accelerator lattice and for each position in phase space, there is a stable spin axis. If the spin of a proton is initially aligned along this direction, it will remain stable, if it isn't, it will precede in a cone around this direction. Finding the stable spin cone corresponding to a given phase space distribution of the particles in the beam is particularly important at injection, since we should make it coincide with the spin cone of the injected beam.

On the closed orbit the spin tune for a polarized particle is the number of spin precesions per turn in an accelerator. It is measured by the quantity $G \gamma$, and increases with the energy of the beam. When, during the acceleration cycle, the spin tune crosses some specific values, spindepolarizing resonances appear. Siberian snakes in the lattice make the spin tune independent of energy. For RHIC, with two Siberian snakes, the spin is made equal to one. This means that the spin pattern should remain identical and repeat at each turn.

To calculate the stable spin axis direction, SPINK uses the method of Stroboscopic Average [6], as implemented in the code SPRINT [7]. A number of protons with different initial spin directions are tracked for a certain number of turns, then only one proton with the average spin is tracked back to reach the initial phase space location. The spin at this starting point, averaged over the multiple passages through the same location in the lattice, is applied to the proton, and the process continues with forward and backward tracking until the process eventually converges.

Fig. 3 shows an example of calculated stable spin axis in RHIC for very small emittance of the beam, at injection ( $G \gamma=41.5$ ). The two snakes were set for spin rotation of $180^{\circ}$ and $100^{\circ}$, respectively, around several settings of mutual perpendicular axes. This arrangement produces a stable spin axis on a cone of semi-aperture $\theta=$ $40^{\circ}$. For each snake axis orientation there is a corresponding angle $\phi$ for the stable spin axis.

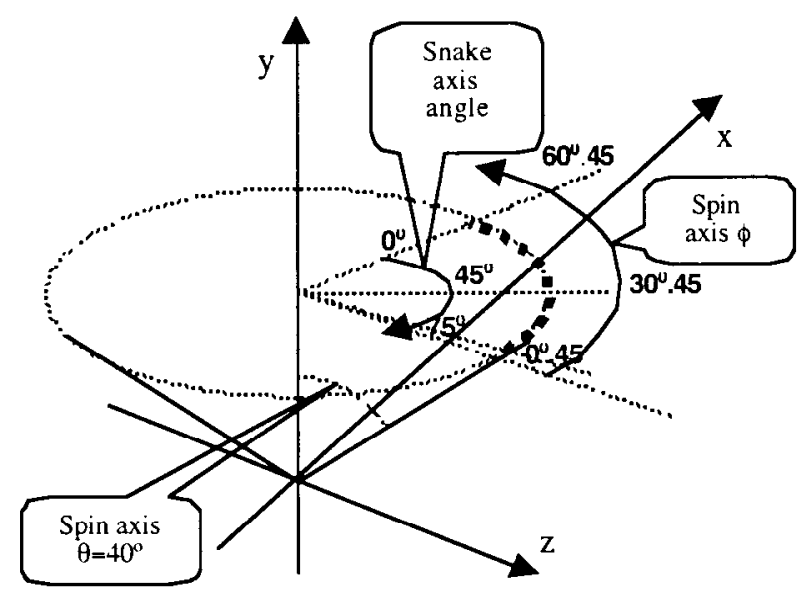

Figure 3: stable spin axis for spin rotation $180^{\circ}$ and $100^{\circ}$ in two snakes around different axis settings 
For the same example, Fig. 4 shows the pattern of the three components of the stable spin axis along the circumference of RHIC. The vertical component of the spin axis is flipped at the two locations of the snakes.

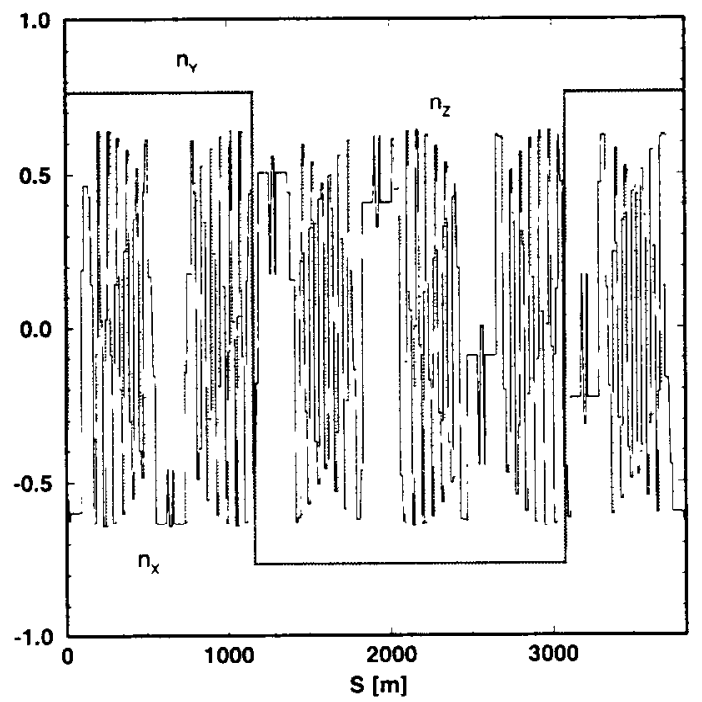

Figure 4. Stable spin axis components in RHIC. Two snakes with spin rotation of $180^{\circ}$ and $100^{\circ}$, and axes at $+45^{\circ}$ and $-45^{\circ}$, respectively.

\section{MAP-GENERATED MATRICES FOR HELICAL SNAKES}

RHIC will be equipped with helical Siberian snakes and Spin rotators. SPINK has three ways to treat helical devices of this kind (i) Synthetic Snakes, (ii) Analytical Snakes, and (iii) Map Snakes.

In the first mode, snakes and rotators are represented for orbit tracking as thin passive elements (MAD Markers), and for spin tracking by a matrix that effectively represents a rotation of the spin vector by a given angle and around a given axis.

Analytical helical devices are represented by a formalism that produces the transport of orbit and spin coordinates through the field of helices, expanded to first order [8].

Map helical devices is implemented by transformations up to third order of the coordinates, whose coefficients have been numerically calculated by tracking by numerical integration a distribution of particles in phase space and spin space through magnetic field maps for the actual devices. The first order part of these maps has been made simplectic [9].

For the first mode, input of $S P I N K$ is the spin rotation and axis angles. For the second, values of the nominal field and characteristic angles for the helices are used. For the third, a set of numerical coefficients.

Fig. 5 shows the geometry of a 4 -helix Siberian snake for RHIC [10] and the trajectories of protons used to calculate the transport maps by third-order fitting.

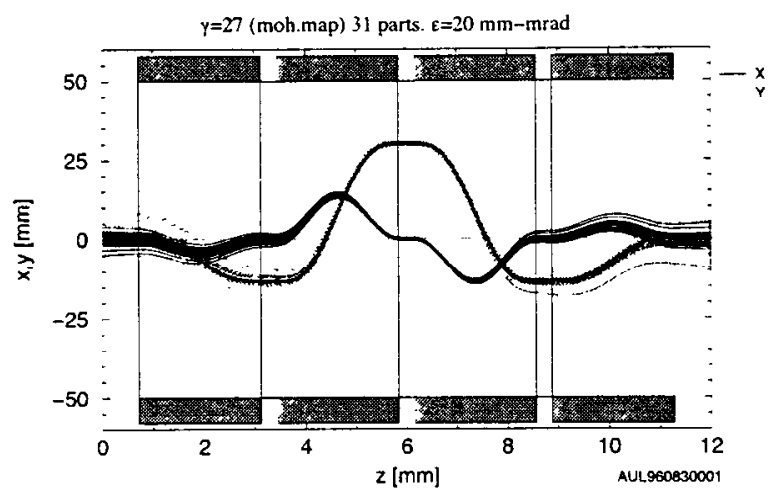

Figure 5: Envelope of trajectories used to calculate $R$ and $T$ orbit and spin maps in a 4-helices Siberian snake.

\section{REFERENCES}

[1] A.U.Luccio in "Trends in Collider Spin Physics", Trieste, Italy. 5-8 Dec. 1995, p.235.

A.U.Luccio. T.Katayama and H. Wu, Proc. of PAC97, Vancouver, BC, Canada, May 12-16, 1997, p.92

[2] T.Roser "The Acceleration of Polarized Protons to High Energies", these Proceedings

[3] H.Grote and F.C.Iselin, "The MAD Program Vers. 8.16" CERN/SL/90-13, Geneva, Switzerland. May, p 26. 1995

[4] J.Niederer, " $Z$ Twiss Tracking Matrix File" BNL MAD Programming Note, Upton, NY, updated March 1999

[5] A.U.Luccio and M.Syphers AGS/RHIC/SN No.068, Upton, NY, Nov. 25, 1997

[6] K.Heinemann and Hoffståtter , Phys.Rev. E54, 4240

[7] D.P.Barber and M.Vogt, Proc. of the EPAC98, Stockholm, Sweden, 22-26 June 1998, p. 1362

[8] E.D.Courant, AGS/RHIC/SN No. 004, Upton, NY, Nov.8 1994. M.J.Syphers AGS/RHIC/SN No.020, Upton, NY. Feb. 1996

[9] A.U.Luccio, "Field Map Generated Matrices for Spin Tracking", Riken-AF-NP-235, Wako, Japan, October 1996

[10] V.I.Ptitsin and Yu.M.Shatunov, in "Proc Third Workshop on Siberian Snakes and Spin Rotators" (A.Luccio and Th.Roser Eds.), BNL-52453, Upton, NY Sept. 12-13. 1994. p. 15. 